\title{
Radiation Effects on Unsteady MHD Free Convective Couette Flow of Heat Generation/Absorbing Fluid
}

\author{
S. Das \\ Department of Mathematics \\ University of Gour Banga \\ Malda 732 103, India
}

\author{
S. K. Guchhait \\ Department of Applied Mathematics \\ Vidyasagar University \\ Midnapore 721 102, India
}

\author{
R. N. Jana \\ Department of Applied \\ Mathematics \\ Vidyasagar University \\ Midnapore 721 102, India
}

\begin{abstract}
Radiation effects on free convection MHD Couette flow of a viscous incompressible heat generating fluid confined between vertical plates have been studied. The governing equations are solved analytically using the Laplace transform technique. The variations of velocity and fluid temperature are presented graphically. It is observed that the velocity decreases with an increase in either magnetic parameter or radiation parameter or generation parameter or Prandtl number. It is also observed that the velocity increases with an increase in either Grashof number or time. An increase in either radiation parameter or Prandtl number leads to fall in the fluid temperature. It is seen that the fluid temperature increases with an increase in either heat generation parameter or time. Further, it is seen that the absolute value of shear stress at the moving plate increases with an increase in either magnetic parameter or radiation parameter while it decreases with an increase in either heat generation parameter or Prandtl number. The rate of heat transfer increases with an increase in either Prandtl number or heat generation parameter or time.
\end{abstract}

Keywords: MHD Couette, free convection, magnetic parameter, radiation, heat generation, Prandtl number and Grashof number.

\section{INTRODUCTION}

Research works in magnetohydrodynamics (MHD) have been advanced significantly during last three decades in natural sciences and engineering disciplines after the pioneer work of Hartmann (Hartmann 1937) in liquid metal duct flow under the influence of a strong external magnetic field. This fundamental investigation has provided basic knowledge for the development of several MHD devices such as MHD pumps, generators, breaks and flow meters. The study of flow for an electrically conducting fluid has many applications in engineering problems such as plasma studies, nuclear reactors, geothermal energy extraction and the boundary layer control in the field of aerodynamics. Recent advances and applications of MHD based microfluidic devices are extensively reviewed in the paper by Qian and Bau [1]. Some of these devices include MHD-based micro-pumps used for producing a mechanical force which sets the fluid into motion; MHD-based microfluidic networks used for transporting fluids and reagents across networks of conduits where the flow control typically requires the use of pumps and valves; MHD-based liquid chromatography used for the separation, purification and detection of various biochemicals. Although, some of these devices are fabricated with low temperature co-fired ceramic tapes, significant heat generation or radiative heat transfer occurs due to the induction of eddy currents in most of these engineering applications [2, 3, 4]. Other examples are, high temperature phenomena or high-power radiation sources commonly encountered in solar physics-particularly in astrophysical studies [5], in combustion applications such as fires, furnaces, IC engines, in nuclear reactions such as in the sun or in nuclear explosions [6], in compressors in ships and in gas flares from petrochemical industry [7]. For air, the contribution of radiation becomes significant when the wall temperature is in the range $6000-10,000 \mathrm{~K}$. This situation is encountered for re-entry space vehicles. Korycki [8] described radiative heat transfer as an important fundamental phenomena existing in practical engineering such as those found in solar radiation in buildings, foundry engineering and solidification processes, die forging, chemical engineering, composite structures applied in industry. Another important feature that usually occurs in electronic devices over a period of continuous usage is the hotness of the surface. This means that a poor design could trap heat generated by the source of the power supply and could incapacitate the efficiency and durability of the systems. Therefore, the efficiency in the functioning of these systems is enhanced when they are subjected to external cooling devices like air conditioners, electric fans, and some others (e.g. laptop computers) inbuilt storage devices that store electrical energy for them to function for sometime even without external source of power supply [9]. The IC components of these electronic systems are thermally coupled to the surrounding via convection and radiation. Radiation has a significant role in heat transfer in low-flow applications where there exists a larger temperature gradient between the components and the surrounding. Couette flow is one of the basic flow in fluid dynamics that refers to the laminar flow of a viscous fluid in the space between two parallel plates, one of which is moving relative to the other. The flow is driven by virtue of viscous drag force acting on the fluid and the applied pressure gradient parallel to the plates. Couette flow is frequently used in physics and engineering to illustrate sheardriven fluid motion. Radiative free convection MHD Couette flows are frequently encountered in many scientific and environmental processes, such as astrophysical flows, heating and cooling of chambers and solar power technology. Heat transfer by simultaneous radiation and convection has applications in numerous technological problems including combustion, furnace design, the design of high temperature gas cooled nuclear reactors, nuclear reactor safety, fluidized bed heat exchanger, fire spreads, solar fans, solar collectors natural convection in cavities, turbid water bodies, photo chemical reactors and many others. Jha[10] has studied the natural convection in unsteady MHD Couette flow. Radiative heat transfer to magnetohydrodynamic Couette flow with variable wall temperature has been investigated by Ogulu and Motsa [11]. Radiation effects on MHD Couette flow with heat transfer between two parallel plates have been examined by Mebine 
[12]. Jha and Ajibade [13] have made an analysis on the free convective flow of heat generating/ absorbing fluid between vertical porous plates with periodic heat input. Jha and Ajibade [14] have studied the unsteady free convective Couette flow of heat generating/absorbing fluid. MHD oscillatory Couette flow of a radiating viscous fluid in a porous medium with periodic wall temperature have been examined by Cookey et al.[15]. Effects of thermal radiation and free convection currents on the unsteady Couette flow between two vertical parallel plates with constant heat flux at one boundary has been studied by Narahari [16]. Deka and Bhattacharya [17] have investigated the unsteady free convective Couette flow of heat generating/absorbing fluid in porous medium. The radiation and free convection effects on a MHD flow through a porous medium between infinite parallel plates with time-dependent suction by have been described by Alagoa et al. [18]. Gbadeyan et al.[19] have discussed the radiation effect on electrohydrodynamic froth flow in vertical channel. The magnetic field and thermal radiation effects on steady hydromagnetic couette flow through a porous channel have been studied by Baoku et al.[20].

In this present paper we investigate the radiation effects on on free convection MHD Couette flow of a viscous incompressible heat generating fluid confined between vertical plates. It is observed that the velocity $u_{1}$ decreases with an increase in either magnetic parameter $M^{2}$ or radiation parameter $R$ or Prandtl number $\operatorname{Pr}$. It is also observed that the velocity $u_{1}(\eta)$ increases with an increase in either heat generation parameter or Grashof number $G r$ or time $\tau$. An increase in either radiation parameter $R$ or Prandtl number $\operatorname{Pr}$ leads to fall in the fluid temperature $\theta$. It is seen that the fluid temperature $\theta$ increases with an increase in either heat generation parameter $\phi$ or time. Further, it is seen that the absolute value of shear stress at the moving plate $\tau_{0}$ increases motion with an increase in either magnetic parameter or radiation parameter while it decreases with an increase in either heat generation parameter or Prandtl number. The rate of heat transfer $-\theta^{\prime}(0)$ increases with an increase in either Prandtl number or $\phi$ or $\tau$.

\section{FORMULATION OF THE PROBLEM AND ITS SOLUTIONS}

Consider the unsteady free convection MHD Couette flow of a viscous incompressible radiative heat generating fluid between two infinite vertical parallel walls separated by a distance $h$. The flow is set up by the buoyancy force arising from the temperature gradient occurring as a result of asymmetric heating of the parallel plates as well as constant motion of one of the plates. Choose a cartesian co-ordinates system with the $x$ - axis along one of the plates in the vertically upward direction and the $y$-axis normal to the plates [See Figure 1.]. Initially, at time $t \leq 0$, the two plates and the fluid are assumed to be at the same temperature $T_{0}$ and stationary. At time $t>0$, the plate at $y=0$ starts moving in its own plane with a velocity $U$ and is heated with temperature $T_{0}$ whereas the plate at $y=h$ is stationary and maintained at a constant temperature $T_{0}$. A uniform magnetic field of strength $B_{0}$ is imposed perpendicular to the plates. It is also assumed that the radiative heat flux in the $x$-direction is negligible as compared to that in the $y$-direction. As the plates are infinite long, the velocity and temperature fields are functions of $y$ and $t$ only.

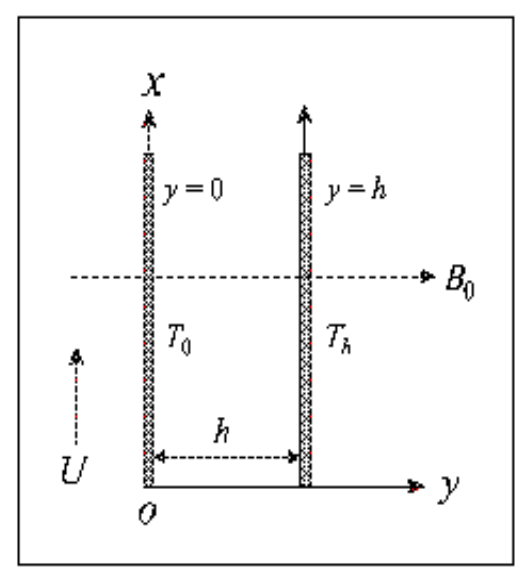

Figure 1. : Geometry of the problem

The Boussinesq approximation is assumed to hold and for the evaluation of the gravitational body force, the density is assumed to depend on the temperature according to the equation of state

$$
\rho=\rho_{0}\left[1-\beta\left(T-T_{h}\right)\right],
$$

where $T$ is the fluid temperature, $\rho$ the fluid density, $\beta$ the coefficient of thermal expansion and $\rho_{0}$ the fluid density at the entrance of the channel.

Then the fully developed flow of a radiating gas is governed by the following set of equations

$$
\begin{aligned}
& \frac{\partial u}{\partial t}=v \frac{\partial^{2} u}{\partial y^{2}}+g \beta\left(T-T_{h}\right)-\frac{\sigma B_{0}^{2}}{\rho} u, \\
& \rho c_{p} \frac{\partial T}{\partial t}=k \frac{\partial^{2} T}{\partial y^{2}}-\frac{\partial q_{r}}{\partial y}-Q_{0}\left(T-T_{h}\right),
\end{aligned}
$$

where $u$ is the velocity in the $y$-direction, $g$ the acceleration due to gravity, $v$ the kinematic coefficient of viscosity, $\rho$ the fluid density, $k$ the thermal conductivity, $c_{p}$ the specific heat at constant pressure, $q_{r}$ the radiative heat flux and $Q_{0}$ a constant.

The initial and the boundary conditions for velocity and temperature distribution are as follows:

$$
\begin{aligned}
& u=0, T=T_{h} \text { for } 0 \leq y \leq h \text { and } t \leq 0, \\
& u=U, T=T_{0} \text { at } y=0 \text { for } t>0, \\
& u=0, T=T_{h} \text { at } y=h \text { for } t>0 .
\end{aligned}
$$

It has been shown by Cogley et al.[21] that in the optically thin limit for a non-gray gas near equilibrium, the following relation holds

$$
\frac{\partial q_{r}}{\partial y}=4\left(T-T_{h}\right) \int_{0}^{\infty} K_{\lambda_{h}}\left(\frac{\partial e_{\lambda p}}{\partial T}\right)_{h} d \lambda,
$$

where $K_{\lambda}$ is the absorption coefficient, $\lambda$ is the wave length, $e_{\lambda p}$ is the Plank's function and subscript ' 0 ' indicates that all quantities have been evaluated at the temperature $T_{h}$ which is the temperature of the plate at time $t \leq 0$. Thus our study is limited to small difference of plate temperature to the fluid temperature.

On use of the equation (5), equation (3) becomes

$$
\rho c_{p} \frac{\partial T}{\partial t}=k \frac{\partial^{2} T}{\partial y^{2}}-4\left(T-T_{h}\right) I-Q_{0}\left(T-T_{h}\right),
$$

where 


$$
I=\int_{0}^{\infty} K_{\lambda_{h}}\left(\frac{\partial e_{\lambda p}}{\partial T}\right)_{h} d \lambda .
$$

Greif et al. [22] showed that, for an optically thin limit, the fluid does not absorb its own emitted radiation, this means that there is no self-absorption, but the fluid does absorb radiation emitted by the boundaries.

We introduce non-dimensional variables

$$
\eta=\frac{y}{h}, \tau=\frac{v t}{h^{2}}, u_{1}=\frac{u}{U}, \theta=\frac{T-T_{h}}{T_{0}-T_{h}} .
$$

On the use of (8), equations (2) and (6) become

$$
\begin{aligned}
& \frac{\partial u_{1}}{\partial \tau}=\frac{\partial^{2} u_{1}}{\partial \eta^{2}}+\operatorname{Gr} \theta-M^{2} u_{1}, \\
& \operatorname{Pr} \frac{\partial \theta}{\partial \tau}=\frac{\partial^{2} \theta}{\partial \eta^{2}}-(R+\phi) \theta,
\end{aligned}
$$

where $M^{2}=\frac{\sigma B_{0}^{2} h^{2}}{\rho v}$ is the magnetic parameter, $R=\frac{4 I h^{2}}{k}$ the radiation parameter, $\phi=\frac{Q_{0} h^{2}}{k}$ the heat generation parameter, $G r=\frac{g \beta\left(T_{0}-T_{h}\right) h^{2}}{v U}$ the Grashof number and $\operatorname{Pr}=\frac{\rho v c_{p}}{k}$ the Prandtl number.

The corresponding initial and boundary conditions for $u_{1}$ and $\theta$ are

$$
\begin{aligned}
& u_{1}=0, \theta=0 \text { for } 0 \leq \eta \leq 1 \text { and } \tau \leq 0, \\
& u_{1}=1, \theta=1 \text { at } \eta=0 \text { for } \tau>0, \\
& u_{1}=0, \theta=0 \text { at } \eta=1 \text { for } \tau>0 .
\end{aligned}
$$

Taking Laplace transformation of the equations (9) and (10), we get

$$
\begin{aligned}
& s \bar{u}_{1}=\frac{d^{2} \bar{u}_{1}}{d \eta^{2}}+G r \bar{\theta}-M^{2} \bar{u}_{1}, \\
& \operatorname{Pr} s \bar{\theta}=\frac{d^{2} \bar{\theta}}{d \eta^{2}}-(R+\phi) \bar{\theta},
\end{aligned}
$$

where

$$
\bar{u}_{1}(\eta, s)=\int_{0}^{\infty} u_{1}(\eta, s) e^{-s \tau} d \tau \text { and } \bar{\theta}(\eta, s)=\int_{0}^{\infty} \theta(\eta, s) e^{-s \tau} d \tau \text {. }
$$

The corresponding boundary conditions for $\bar{u}_{1}$ and $\bar{\theta}$ are

$$
\begin{aligned}
& \bar{u}_{1}(0, s)=\frac{1}{s}, \bar{\theta}(0, s)=\frac{1}{s}, \\
& \bar{u}_{1}(1, s)=0, \bar{\theta}(1, s)=0 .
\end{aligned}
$$

The solution of the equations (13) and (12) subject to the boundary conditions (15) are easily obtained and are given by

$$
\begin{aligned}
\bar{\theta}(\eta, s) & =\frac{1}{s} \frac{\sinh \sqrt{\operatorname{Pr}(s+\alpha)}(1-\eta)}{\sinh \sqrt{\operatorname{Pr}(s+\alpha)}}, \\
\bar{u}_{1}(\eta, s) & =\frac{1}{s} \frac{\sinh \sqrt{s+M^{2}}(1-\eta)}{\sinh \sqrt{s+M^{2}}} \\
& +\frac{G r}{(\operatorname{Pr}-1) s(s+b)}\left[\frac{\sinh \sqrt{s+M^{2}}(1-\eta)}{\sinh \sqrt{s+M^{2}}}\right. \\
& \left.-\frac{\sinh \sqrt{\operatorname{Pr}(s+\alpha)(1-\eta)}}{\sinh \sqrt{\operatorname{Pr}(s+\alpha)}}\right],
\end{aligned}
$$

where

$$
\alpha=\frac{R+\phi}{P r} \text { and } b=\frac{\alpha P r-M^{2}}{P r-1} .
$$

The inverse transforms of (16) and (17) give the solution for the temperature and velocity field distributions as

$$
\begin{aligned}
& \theta(\eta, \tau)= \begin{cases}\frac{\sinh \sqrt{\alpha \operatorname{Pr}}(1-\eta)}{\sinh \sqrt{\alpha \operatorname{Pr}}}-2 \pi \sum_{n=1}^{\infty} \frac{n e^{-\left(n^{2} \pi^{2} / P r+\alpha\right) \tau}}{n^{2} \pi^{2}+\alpha \operatorname{Pr}} \sin n \pi \eta & \text { for } \operatorname{Pr} \neq 1, \\
\frac{\sinh \sqrt{R+\phi}(1-\eta)}{\sinh \sqrt{R+\phi}}-2 \pi \sum_{n=1}^{\infty} \frac{n e^{-\left(n^{2} \pi^{2}+R+\phi\right) \tau}}{n^{2} \pi^{2}+R+\phi} \sin n \pi \eta & \text { for } \operatorname{Pr}=1\end{cases}
\end{aligned}
$$

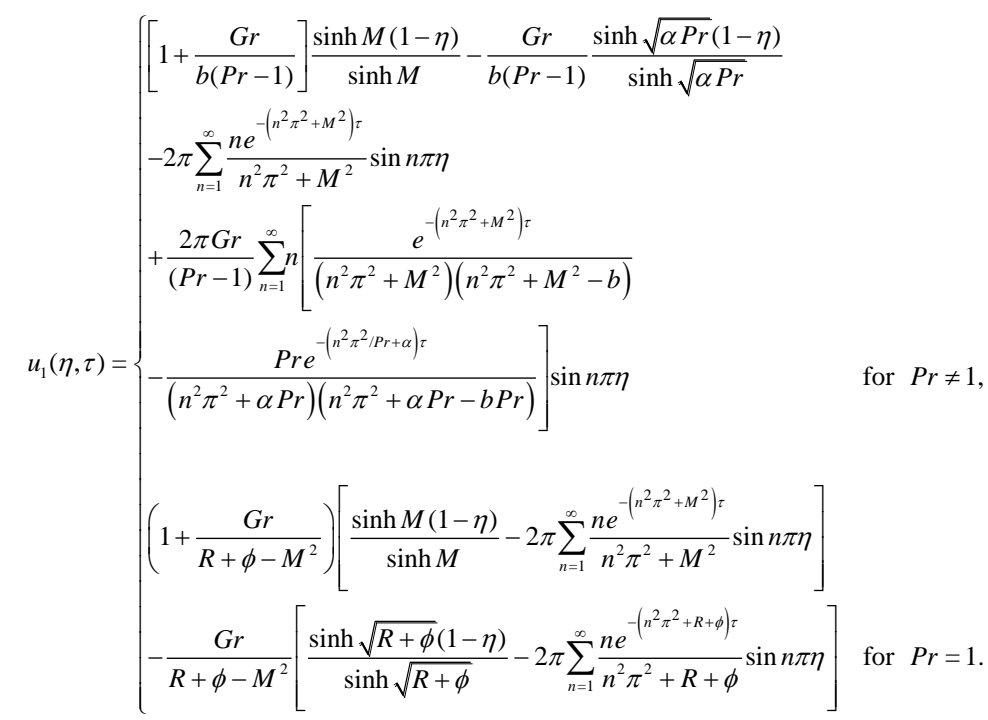

where $\alpha$ and $b$ are given by (18). 
For steady state, the temperature distribution and velocity field are obtained as

$$
\theta(\eta, \tau)= \begin{cases}\frac{\sinh \sqrt{\alpha \operatorname{Pr}}(1-\eta)}{\sinh \sqrt{\alpha \operatorname{Pr}}} & \text { for } \operatorname{Pr} \neq 1, \\ \frac{\sinh \sqrt{R+\phi}(1-\eta)}{\sinh \sqrt{R+\phi}} & \text { for } \operatorname{Pr}=1\end{cases}
$$

$$
u_{1}(\eta, \tau)= \begin{cases}{\left[1+\frac{G r}{b(P r-1)}\right] \frac{\sinh M(1-\eta)}{\sinh M}} & \text { for } \operatorname{Pr} \neq 1, \\ -\frac{G r}{b(P r-1)} \frac{\sinh \sqrt{\alpha \operatorname{Pr}}(1-\eta)}{\sinh \sqrt{\alpha \operatorname{lP}}} & \\ \left(1+\frac{G r}{R+\phi-M^{2}}\right) \frac{\sinh M(1-\eta)}{\sinh M} & \text { for } \operatorname{Pr}=1 . \\ -\frac{G r}{R+\phi-M^{2}} \frac{\sinh \sqrt{R+\phi}(1-\eta)}{\sinh \sqrt{R+\phi}} & \end{cases}
$$

As the Prandtl number is a measure of the relative importance of the viscosity and thermal conductivity of the fluid, the case $\operatorname{Pr}=1$ corresponds to those fluids whose momentum and thermal boundary layer thicknesses are of the same order of magnitude. Thus the solution for the velocity field has to be re-derived from equations (9) and (10) when $\operatorname{Pr}=1$.

\section{RESULTS AND DISCUSSION}

We have presented the non-dimensional velocity and temperature for several values of magnetic parameter $M^{2}$, radiation parameter $R$, heat generation parameter $\phi$, Prandtl number $\mathrm{Pr}$, Grashof number $\mathrm{Gr}$ and time $\tau$ in Figures 2-11. Figures 2-7 represent the velocity $u_{1}$ against $\eta$ for several values of $M^{2}, R, \phi, P r, G r$ and $\tau$. It is seen from Figure 2 that the velocity $u_{1}$ decreases with an increase in magnetic parameter $M^{2}$. This observation can be explained by the fact that as $M$ increases, the Lorentz force which opposes the flow, increases and leads to enhanced deceleration of the flow. Figure 3 reveals that the velocity $u_{1}$ decreases with an increase in radiation parameter $R$. This shows that there is a fall in velocity in the presence of high radiation. It is seen from Figure 4 that the velocity $u_{1}$ decreases with an increase in heat generation parameter $\phi$. As $\phi$ increases, heat absorbing capacity of the fluid increases which decreases fluid temperature and hence the fluid velocity. An increase in Prandtl number $\operatorname{Pr}$ leads to decrease the velocity $u_{1}$ near the moving plate and reverse result near stationary plate shown in Figure 5. Physically, this is true because the increase in the Prandtl number is due to increase in the viscosity of the fluid which makes the fluid thick and hence causes a decrease in the velocity of the fluid. It is observed from Figure 6 that an increase in $G r$ leads to fall in the values of velocity $u_{1}$. An increase in Grashof number leads to an increase in velocity, this is because, increase in Grashof number means more heating and less density. It is seen from Figure 7 that the velocity $u_{1}$ increases with an increase in time $\tau$. It is seen from Figure 8 that the temperature $\theta$ decreases as the radiation parameter $R$ increases. This is expected, since the effect of radiation is to decrease the rate of energy transport to the fluid, thereby decreasing the temperature of the fluid. It is seen from Figure 9 that the temperature $\theta$ decreases as the heat generation parameter $\phi$ increases. This result agrees with expectations, as $\phi$ increases, heat absorbing capacity of the fluid increases and hence the fluid temperature decreases. It is observed from Figure 10 that the temperature $\theta$ decreases with an increase in Prandtl number $P r$. This implies that an increase in Prandtl number leads to fall the thermal boundary layer flow. This is because fluids with large $P r$ have low thermal diffusivity which causes low heat penetration resulting in reduced thermal boundary layer. Figure 11 shows that the temperature $\theta$ increases with an increase in time $\tau$. It is observed from Figures 8-11 that temperature decreases gradually from highest value on the moving plate to a zero value on the stationary plate.

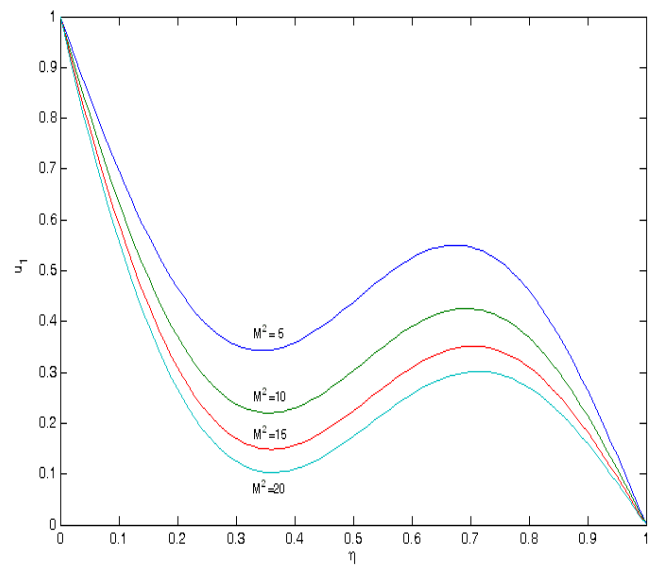

Figure 2. Velocity profiles for $M^{2}$ when $R=2, \phi=2$, $\operatorname{Pr}=0.025, G r=5$ and $\tau=0.0005$.

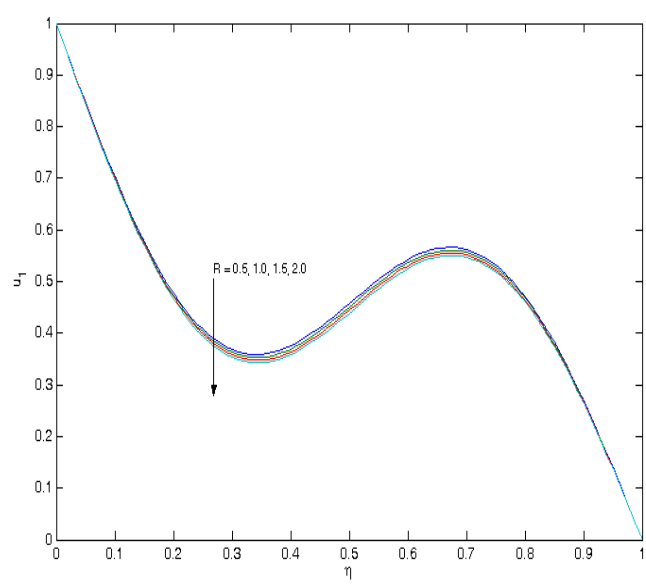

Figure 3. Velocity profiles for $R$ when $M^{2}=5, \phi=2$, $G r=5, P r=0.025$ and $\tau=0.0005$. 


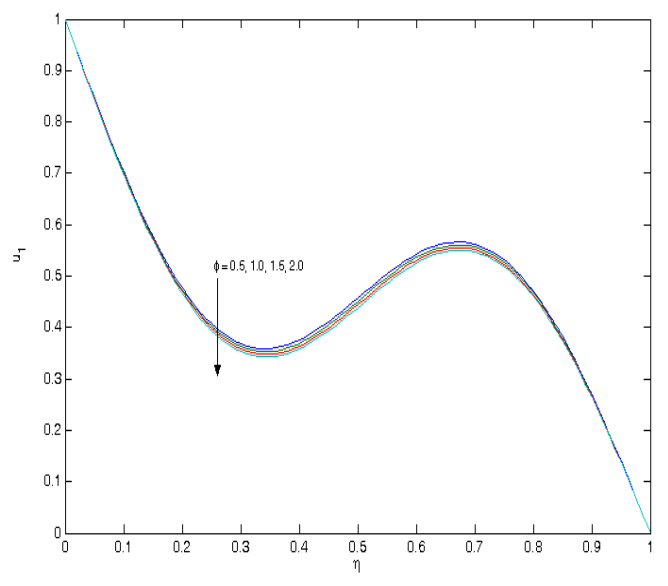

Figure 4. Velocity profiles for $\phi$ when $M^{2}=5, R=2$, $G r=5, P r=0.025$ and $\tau=0.0005$.

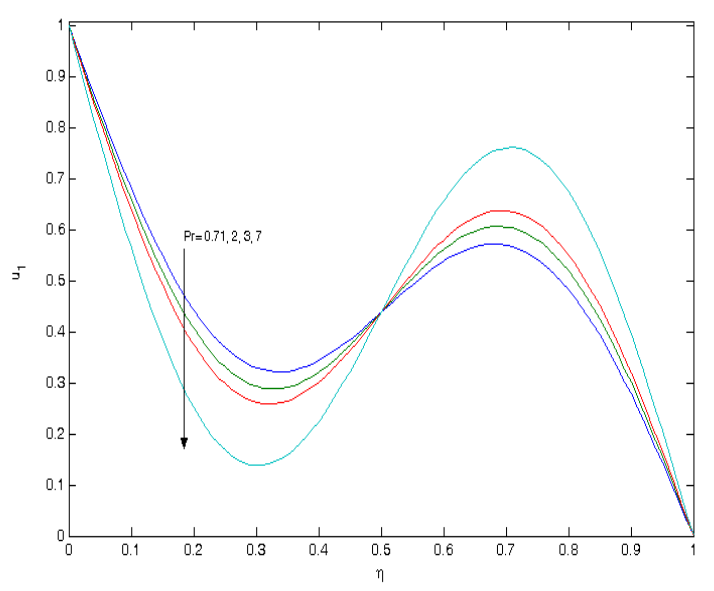

Figure 5. Velocity profiles for $\operatorname{Pr}$ when $M^{2}=5, R=2$, $G r=5, \phi=2$ and $\tau=0.0005$.

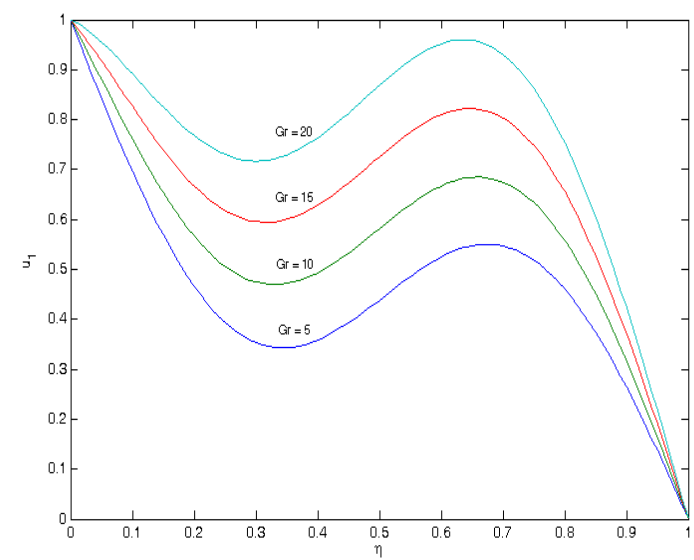

Figure 6. Velocity profiles for $G r$ when $M^{2}=5, R=2$, $\phi=2, \operatorname{Pr}=0.025$ and $\tau=0.0005$.

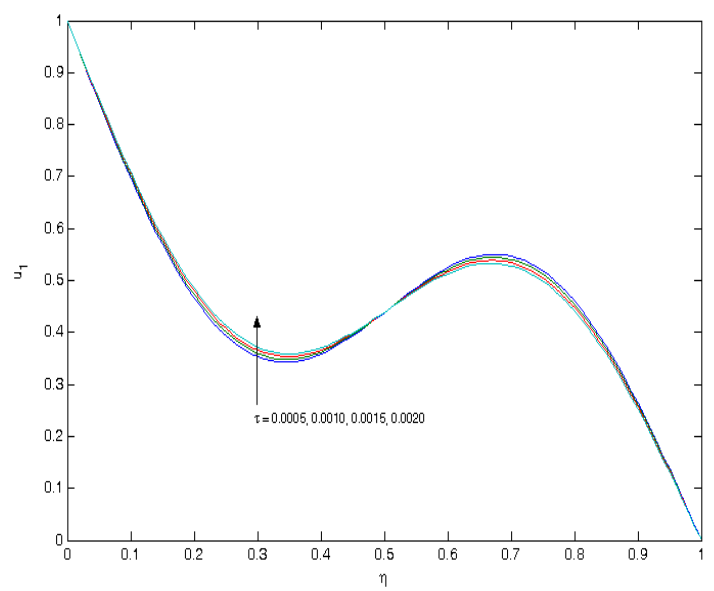

Figure 7. Velocity profiles for time $\tau$ when $M^{2}=5$, $R=2, G r=5, \phi=2$ and $P r=0.025$.

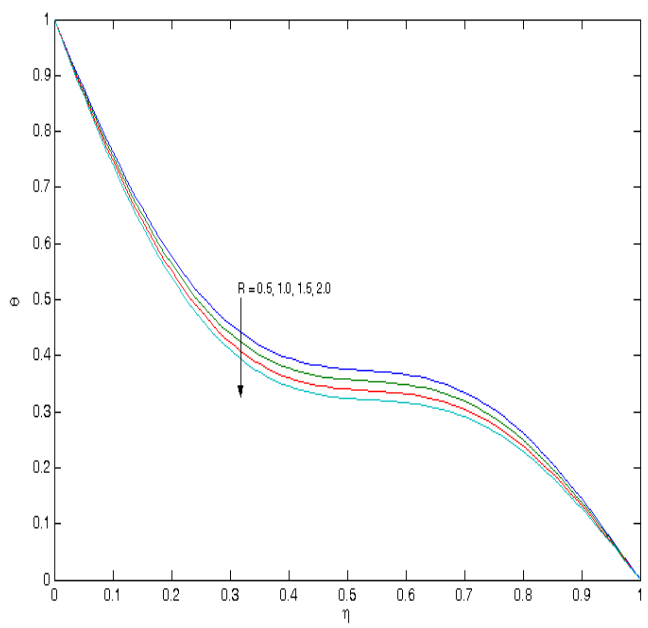

Figure 8. Temperature profiles for $R$ when $P r=0.025$, $\phi=2$ and $\tau=0.0005$.

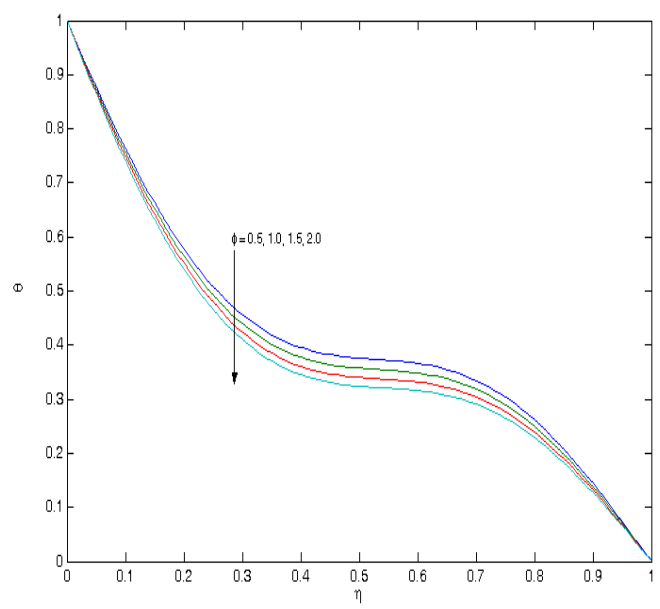

Figure 9. Temperature profiles for $\phi$ when $R=\mathbf{2}$, $\operatorname{Pr}=0.025$ and $\tau=0.5$. 


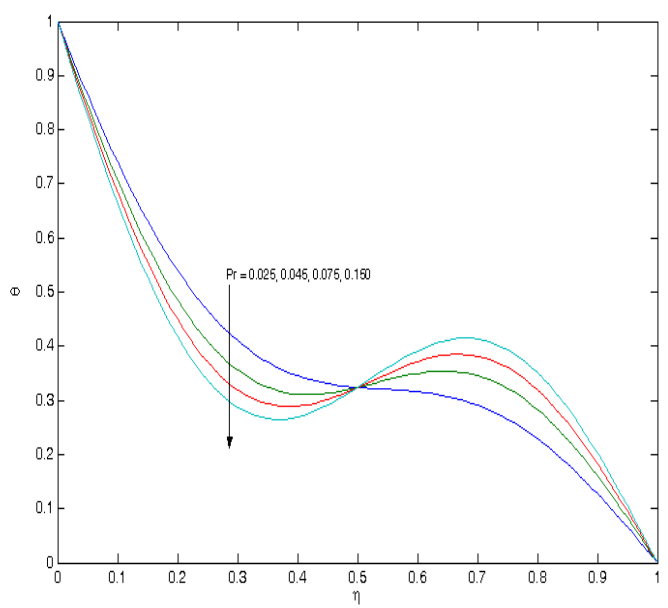

Figure 10. Temperature profiles for $\operatorname{Pr}$ when $R=2, \phi=4$ and $\tau=0.2$.

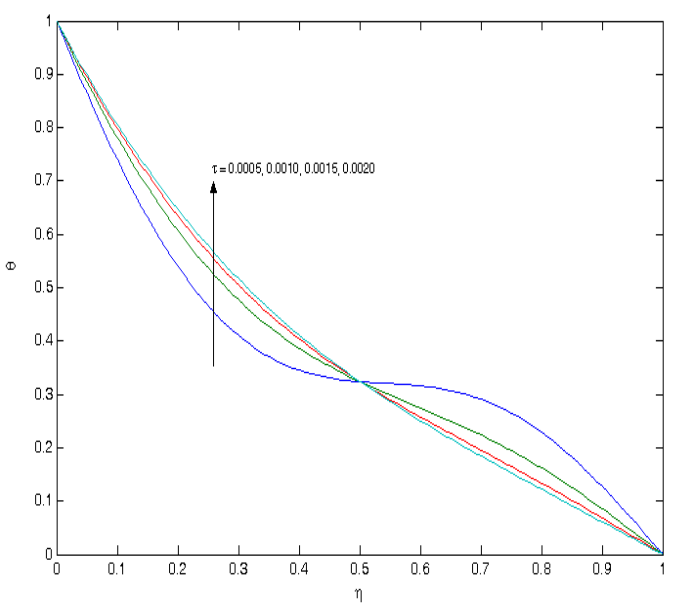

Figure 11. Temperature profiles for $\tau$ when $\operatorname{Pr}=\mathbf{0 . 0 2 5}$, $\boldsymbol{R}=\mathbf{2}$ and $\phi=2$.

From the temperature field, we now study the rate of heat

Table 1. Rate of heat transfer $-10^{-1} \theta^{\prime}(0)$ at the moving plate $\eta=0$.

\begin{tabular}{|l|l|c|c|c|c|c|c|c|c|}
\hline & \multicolumn{3}{|c|}{$R$} & \multicolumn{3}{c|}{ Pr } & \multicolumn{3}{c|}{$\tau$} \\
\hline$\phi$ & 1 & 2 & 3 & 0.71 & 2 & 7 & 0.0020 & 0.0025 & 0.0030 \\
\hline 0.5 & 0.34897 & 0.36921 & 0.38735 & 0.36921 & 0.25677 & 0.19817 & 0.40746 & 0.40060 & 0.39395 \\
1.0 & 0.35938 & 0.37851 & 0.39578 & 0.37851 & 0.26797 & 0.21016 & 0.41667 & 0.40982 & 0.40318 \\
1.5 & 0.36921 & 0.38735 & 0.40384 & 0.38735 & 0.27867 & 0.22162 & 0.42542 & 0.41858 & 0.41195 \\
2.0 & 0.37851 & 0.39578 & 0.41156 & 0.39578 & 0.28891 & 0.23261 & 0.43375 & 0.42693 & 0.42031 \\
\hline
\end{tabular}

From the physical point of view, it is necessary to know the shear stress at the plate $\eta=0$. The non-dimensional shear stress at the plate $\eta=0$ is obtained as: transfer the plate $\eta=0$, which is given in non-dimensional form as

$$
-\theta^{\prime}(0)= \begin{cases}\sqrt{\alpha \operatorname{Pr}} \operatorname{coth} \sqrt{\alpha \operatorname{Pr}} & \\ +2 \pi^{2} \sum_{n=1}^{\infty} \frac{n^{2} e^{-\left(n^{2} \pi^{2} / P r+\alpha\right) \tau}}{n^{2} \pi^{2}+\alpha P r} & \text { for } \operatorname{Pr} \neq 1, \\ \sqrt{R+\phi} \operatorname{coth} \sqrt{R+\phi} & \\ +2 \pi^{2} \sum_{n=1}^{\infty} \frac{n^{2} e^{-\left(n^{2} \pi^{2}+R+\phi\right) \tau}}{n^{2} \pi^{2}+R+\phi} & \text { for } \operatorname{Pr}=1\end{cases}
$$

Numerical results of the rate of heat transfer $-\theta^{\prime}(0)$ at the moving plate $(\eta=0)$ against heat generation parameter $\phi$ are presented in the Table 1 for various values of the radiation parameter $R$, Prandtl number $\operatorname{Pr}$ and time $\tau$. Table 1 shows that for fixed values of $\phi$, the rate of heat transfer $-\theta^{\prime}(0)$ increases with an increase $R$ while it decreases with increase in either Prandtl number $\operatorname{Pr}$ or time $\tau$. This may be explained by the fact that frictional forces become dominant with increasing values of $P r$ and hence yield greater heat transfer rates. Further, it is seen that for fixed value of $R, P r$ and $\tau$, the rate of heat transfer $-\theta^{\prime}(0)$ increases with an increase in generation parameter $\phi$. The negative value of $\theta^{\prime}(0)$ physically explains that there is heat flow from the plate. 


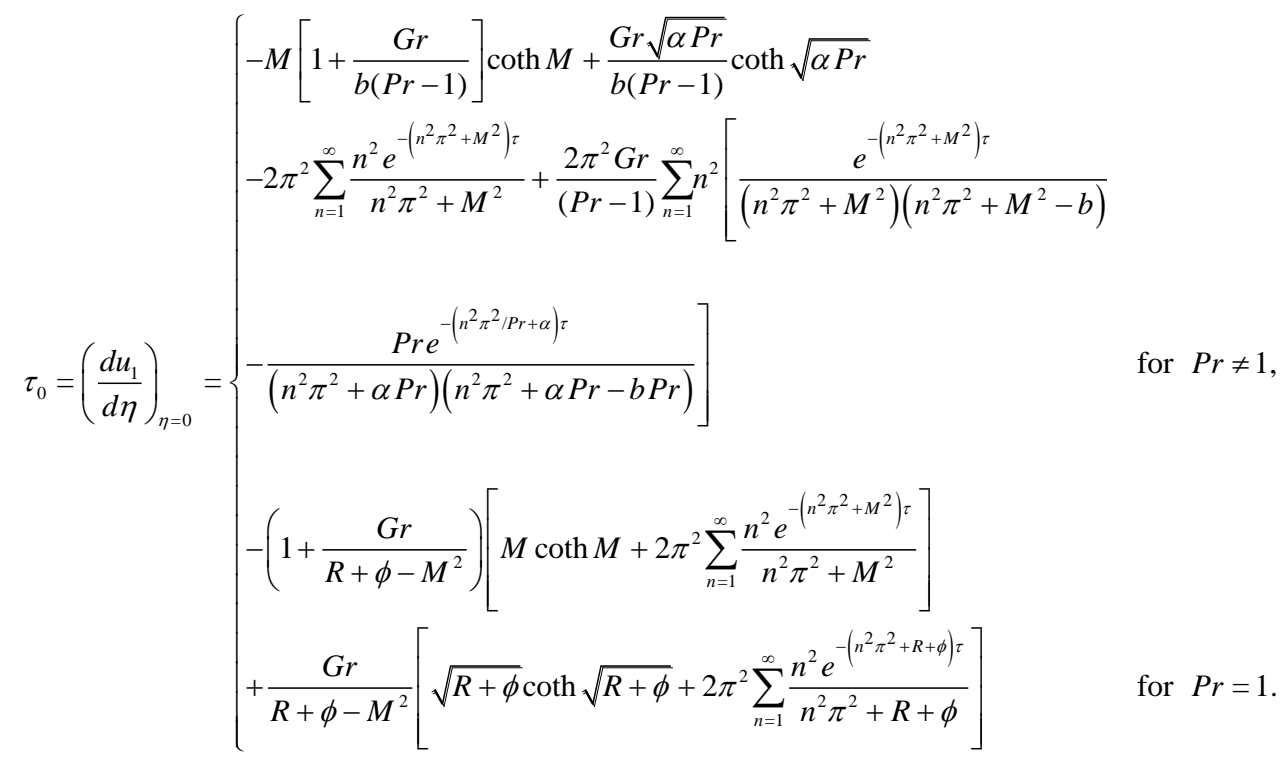

Numerical results of the non-dimensional shear stress $\tau_{0}$ at the lower $(\eta=0)$ are presented in Figures.12-16 against magnetic parameter $M^{2}$ for various values of radiation parameter $R$, heat generation parameter $\phi$, Prandtl number $\mathrm{Pr}$, Grashof number $G r$ and time $\tau$. Figure 12 shows that the shear stress $\tau_{0}$ decreases with an increase in magnetic parameter $M^{2}$ for fixed values of $R, \phi, P r, G r$ and $\tau$. It is seen from Figures.13-17 that for for fixed values of $M^{2}$, the shear stress $\tau_{0}$ decreases with an increase in either radiation parameter $R$ or heat generation parameter $\phi$ or Prandtl number $P r$ while it increases with an increase in either Grashof number $G r$ or time $\tau$. These results are in agrement with the fact that the velocity decreases with an increase in $R$ or $\phi$ or $\operatorname{Pr}$ while it increases with an increases in $G r$ or $\tau$. A large Prandtl number implies more prominent viscous effects causing an enhanced frictional force.

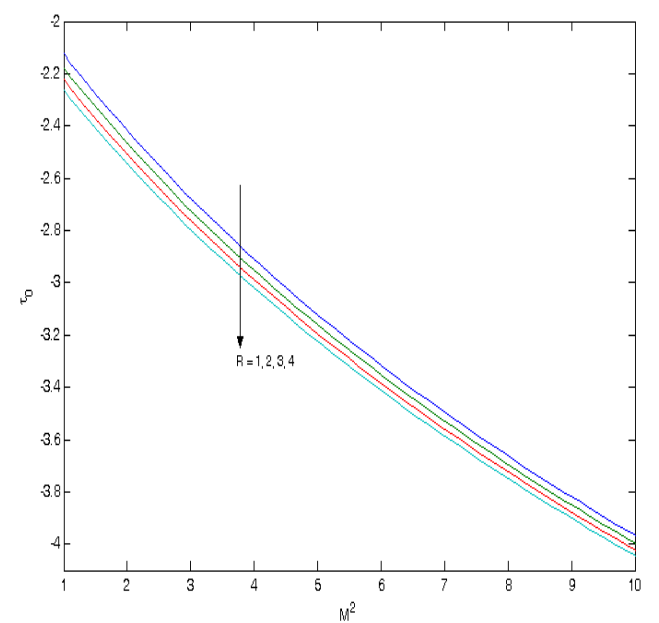

Figure 12. Shear stress $\tau_{0}$ for $R, \phi=2, P r=0.025$ and $\tau=0.0005$.

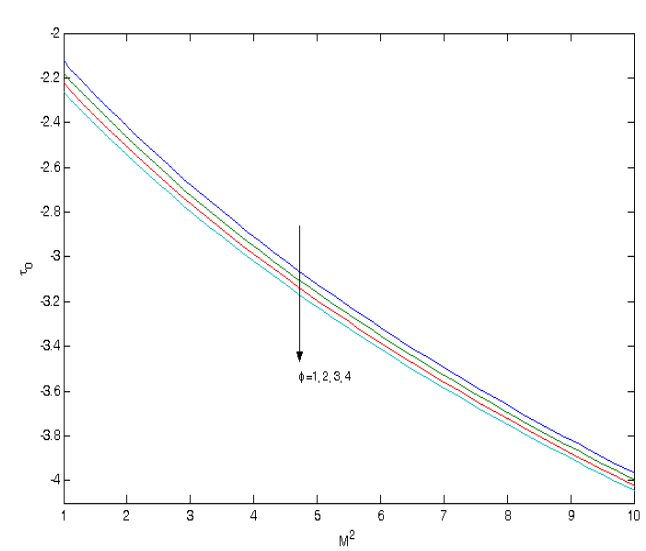

Figure 13. Shear stress $\tau_{0}$ for $\phi$ when $R=2, P r=0.025$ and $\tau=\mathbf{0 . 0 0 0 5}$.

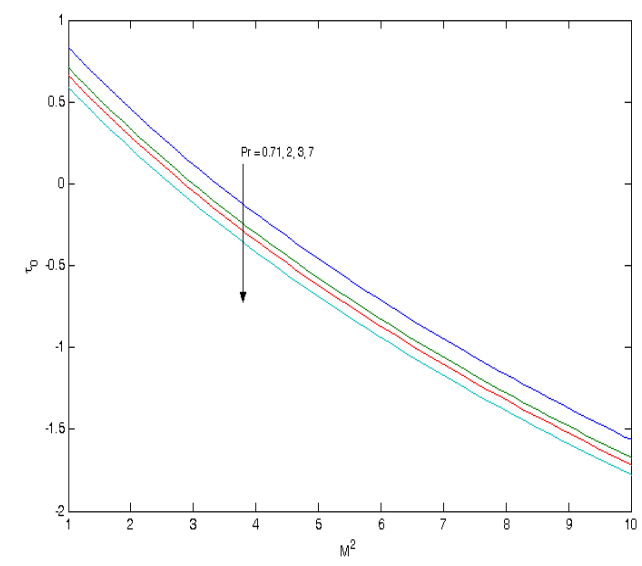

Figure 14. Shear stress $\tau_{0}$ for $\operatorname{Pr}$ when $R=2, \operatorname{Pr}=0.025$ and $\tau=\mathbf{0 . 0 0 0 5}$. 


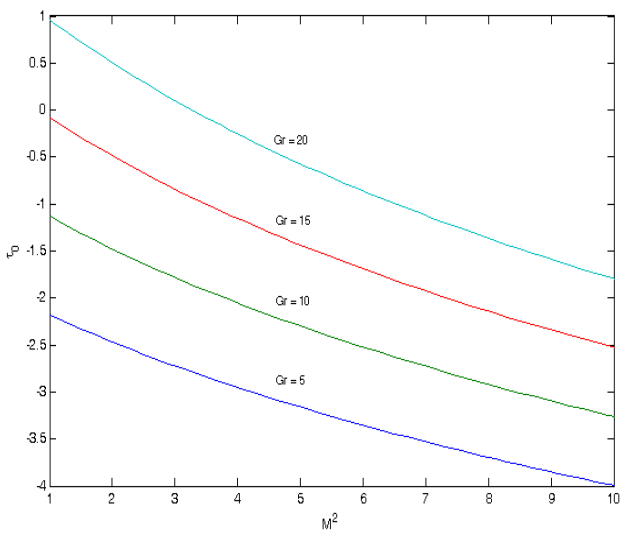

Figure 15. Shear stress $\tau_{0}$ for $G r$ when $R=2, \phi=2$ and $\tau=\mathbf{0 . 0 0 0 5}$.

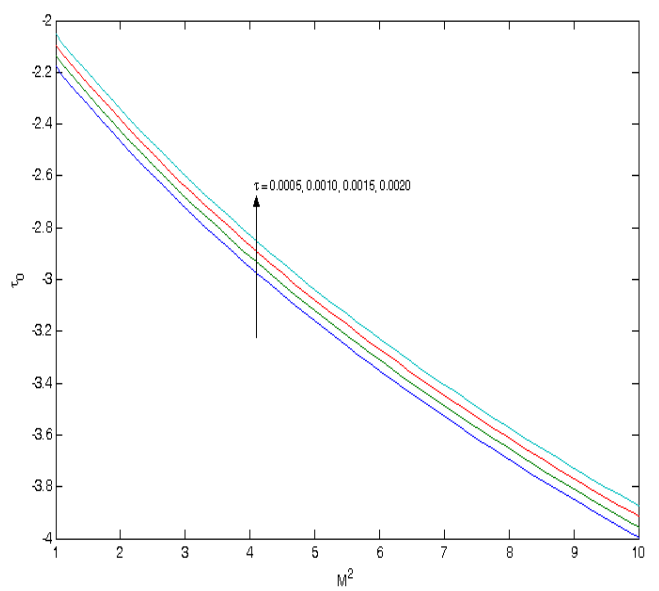

Figure 16. Shear stress $\tau_{0}$ for time $\tau$ when $R=2, \phi=2$ and $P r=0.025$.

The vertical flow rate $Q$ through the channel is given by

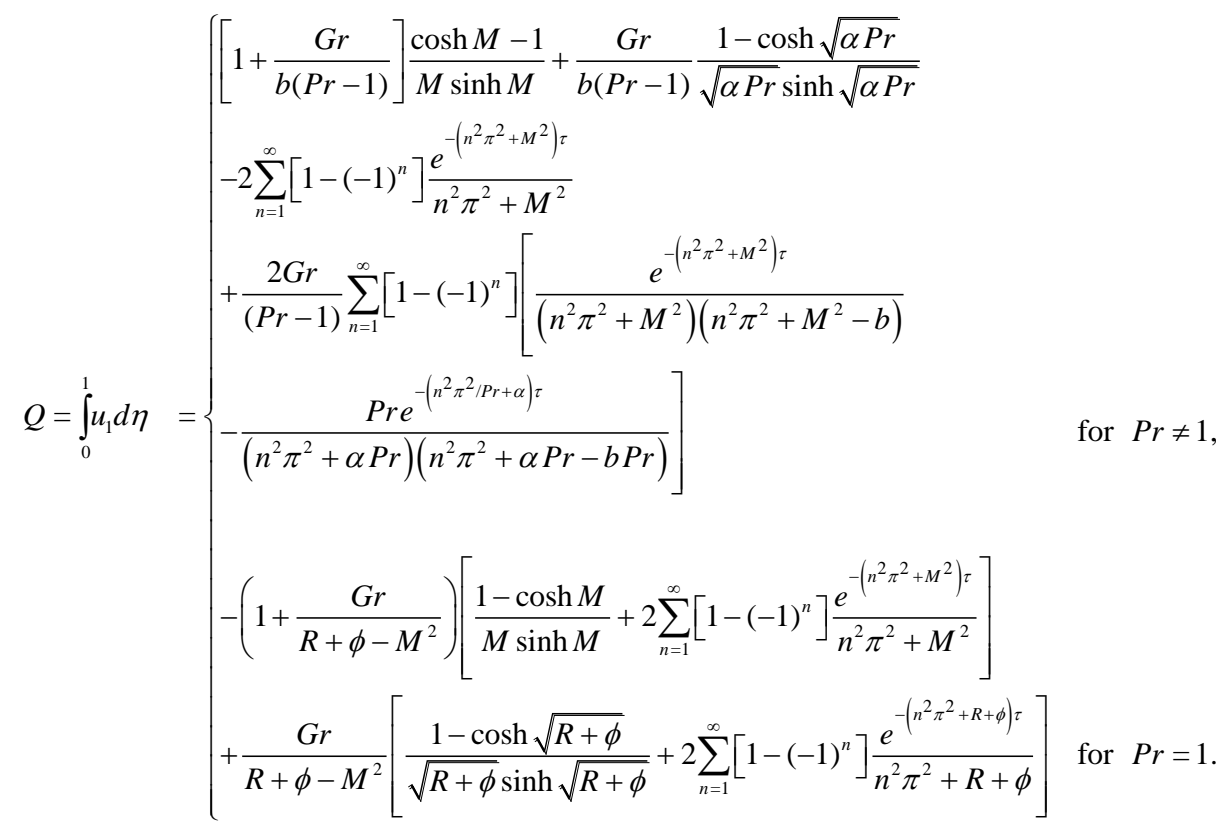

Numerical results of the vertical flow rate $Q$ through the channel against magnetic parameter $M^{2}$ are presented in the Table 2 for various values of the radiation parameter $R$, heat generation parameter $\phi$ and time $\tau$. Table 2 shows that for fixed values of $M^{2}$, the vertical flow rate $Q$ decreases with an increase $R$ or heat generation parameter $\phi$ or time $\tau$.
Further, it is seen that for fixed value of $R, \phi$ and $\tau$, the vertical flow rate $Q$ decreases with an increase in $M^{2}$. We see that the vertical flow rate decreases with increasing radiation parameter and these variations are significant at lower values of time.

Table 2. Vertical flow rate $Q$ with $G r=5$ and $P r=0.71$.

\begin{tabular}{|l|c|c|c|c|c|c|c|c|c|}
\hline & \multicolumn{3}{|c|}{$R$} & \multicolumn{3}{c|}{$\phi$} & \multicolumn{3}{c|}{$\tau$} \\
\hline$M^{2}$ & 1 & 2 & 3 & 0.5 & 1 & 1.5 & 0.005 & 0.0010 & 0.0015 \\
\hline 5 & 0.42515 & 0.41762 & 0.31790 & 0.42938 & 0.42515 & 0.42124 & 0.41762 & 0.41949 & 0.42128 \\
10 & 0.33069 & 0.32505 & 0.32017 & 0.33386 & 0.33069 & 0.32777 & 0.32505 & 0.32692 & 0.32870 \\
15 & 0.27405 & 0.26954 & 0.26564 & 0.27657 & 0.27405 & 0.27171 & 0.26954 & 0.27140 & 0.27317 \\
20 & 0.23622 & 0.23247 & 0.22922 & 0.23833 & 0.23622 & 0.23428 & 0.23247 & 0.23432 & 0.23608 \\
\hline
\end{tabular}


The critical Grashof number for which there is no flow reversal near the plates $\eta=0$ and $\eta=1$ are respectively given by

$$
\begin{aligned}
G r_{0}= & \frac{M \operatorname{coth} M+2 \pi^{2} \sum_{n=1}^{\infty} \frac{n^{2} e^{-\left(n^{2} \pi^{2}+M^{2}\right) \tau}}{n^{2} \pi^{2}+M^{2}}}{-\frac{M}{b(P r-1)} \operatorname{coth} M+\frac{\sqrt{\alpha P r}}{b(P r-1)} \operatorname{coth} \sqrt{\alpha P r}+\frac{2 \pi^{2}}{(P r-1)} \sum_{n=1}^{\infty} n^{2} a_{n}}, \\
G r_{1}= & \frac{M \operatorname{cosech} M+2 \pi^{2} \sum_{n=1}^{\infty} \frac{n^{2}(-1)^{n} e^{-\left(n^{2} \pi^{2}+M^{2}\right) \tau}}{n^{2} \pi^{2}+M^{2}}}{-\frac{M}{b(P r-1)} \operatorname{cosech} M+\frac{\sqrt{\alpha P r}}{b(P r-1)}} \operatorname{cosech} \sqrt{\alpha P r}+\frac{2 \pi^{2}}{(P r-1)} \sum_{n=1}^{\infty} n^{2}(-1)^{n} a_{n}
\end{aligned}
$$

where

$$
a_{n}=\left[\frac{e^{-\left(n^{2} \pi^{2}+M^{2}\right) \tau}}{\left(n^{2} \pi^{2}+M^{2}\right)\left(n^{2} \pi^{2}+M^{2}-b\right)}-\frac{\operatorname{Pr} e^{-\left(n^{2} \pi^{2} / P r+\alpha\right) \tau}}{\left(n^{2} \pi^{2}+\alpha \operatorname{Pr}\right)\left(n^{2} \pi^{2}+\alpha \operatorname{Pr}-b P r\right)}\right] .
$$

The values of the critical Grashof number $G r_{0}$ at the moving plate $\eta=0$ and $G r_{1}$ at the stationary plate $\eta=1$ due to the flow are entered in the Tables 3 and 4 for various values of the radiation parameter $R$, heat generation parameter $\phi$ and time $\tau$ against magnetic parameter $M^{2}$. It is seen from the Table 3 that both the critical Grashof numbers $G r_{0}$ and $G r_{1}$ due to the flow increases with increase in either $R$ or $\phi$ while it decreases with increase in time $\tau$. On the other hand, with increase in the magnetic parameter $M^{2}, G r_{0}$ increases for

\begin{tabular}{|c|c|c|c|c|c|c|c|c|c|}
\hline & \multicolumn{3}{|c|}{$R$} & \multicolumn{3}{|c|}{$\phi$} & \multicolumn{3}{|c|}{$\tau$} \\
\hline$M^{2}$ & 1 & 2 & 3 & 0.5 & 1 & 1.5 & 0.005 & 0.0010 & 0.0015 \\
\hline 5 & 0.18758 & 0.19555 & 0.21017 & 0.18343 & 0.18758 & 0.19162 & 0.19555 & 0.18541 & 0.17639 \\
\hline 10 & 0.26678 & 0.27704 & 0.28677 & 0.26143 & 0.26678 & 0.27198 & 0.27704 & 0.26512 & 0.25451 \\
\hline 15 & 0.34161 & 0.35372 & 0.36517 & 0.33528 & 0.34161 & 0.34775 & 0.35372 & 0.34030 & 0.32831 \\
\hline 20 & 0.41350 & 0.42718 & 0.44009 & 0.40633 & 0.41350 & 0.42044 & 0.42718 & 0.41241 & 0.39921 \\
\hline
\end{tabular}
fixed values of $R, \phi$ and $\tau$.

Table 3. Critical Grashof number $10^{-2} G r_{0}$ at the plate $\eta=0$ with $\operatorname{Pr}=0.71$.

Table 4. Critical Grashof number $10^{-2} G r_{1}$ at the plate $\eta=1$ with $P r=0.71$.

\begin{tabular}{|l|c|c|c|c|c|c|c|c|c|}
\hline & \multicolumn{3}{|c|}{$R$} & \multicolumn{3}{c|}{$\phi$} & \multicolumn{3}{c|}{$\tau$} \\
\hline$M^{2}$ & 1 & 2 & 3 & 0.5 & 1 & 1.5 & 0.005 & 0.0010 & 0.0015 \\
\hline 5 & 0.18631 & 0.20678 & 0.22505 & 0.17643 & 0.18631 & 0.19643 & 0.20678 & 0.17224 & 0.14170 \\
10 & 0.31998 & 0.35899 & 0.40062 & 0.30141 & 0.31998 & 0.33917 & 0.35899 & 0.30600 & 0.25910 \\
15 & 0.46915 & 0.53244 & 0.60144 & 0.43948 & 0.46915 & 0.50012 & 0.53244 & 0.45673 & 0.38964 \\
20 & 0.63531 & 0.73001 & 0.83573 & 0.59166 & 0.63531 & 0.68137 & 0.73001 & 0.62654 & 0.53474 \\
\hline
\end{tabular}

\section{SINGLE VERTICAL PLATE}

In the limit $h \rightarrow \infty$, that is, when one of the plates $(\eta=1)$ is placed at an infinite distance, then the problem is reduced to the flow past an vertical plate with variable temperature in the presence of heat generation. The equations (21) and (22) become

$$
\begin{aligned}
& \theta(\eta)=e^{-\sqrt{R+\phi} \eta}, \\
& u_{1}(\eta)=e^{-M \eta}+\frac{G r}{R+\phi-M^{2}}\left(e^{-M \eta}-e^{-\sqrt{R+\phi} \eta}\right),
\end{aligned}
$$

\section{CONCLUSION}

The radiation effects on transient natural convection flow confined between two infinite vertical walls have been studied. The dimensionless governing partial differential equations are solved by the usual Laplace transform technique. The effect of different parameters such as radiation parameter, Grashof number, Prandtl number, heat generation parameter and time are studied. It is also observed that the velocity $u_{1}$ increases with an increase in either heat generation parameter or Grashof number $\mathrm{Gr}$ or time $\tau$ for both impulsive motion as well as for accelerated motion. An increase in either radiation parameter $R$ or Prandtl number $\operatorname{Pr}$ leads to fall in the fluid temperature $\theta$. It is seen that the fluid temperature $\theta$ increases with an increase in either heat generation parameter $\phi$ or time. Further, it is seen that the shear stress $\tau_{0}$ at the moving plate increases with an increase in either magnetic parameter or radiation parameter while it decreases with an increase in either heat generation parameter or Prandtl number. The rate of heat transfer $-\theta^{\prime}(0)$ increases with an increase in either Prandtl number or $\phi$ or $\tau$. The vertical flow rate decreases with an increase in radiation parameter. Therefore, we may conclude that the interaction between the radiation, MHD effects, buoyancy forces and the heat generation induced by a vertical motion of the plate can affect the configuration of the flow field significantly. 


\section{REFERENCES}

[1] Qian, S. and Bau, H. H. (2009). Magnetohydrodynamics based microfluidics. Mechanics Research Communications. $36: 10-21$

[2] Lemoff, A. V. and Lee, A. P. B. (2000). An AC magnetohydrodynamic micropump. Sensors and Actuators. 63: $178-185$.

[3] West, J. , Karamata, B., Lillis, B., Gleeson, J. P., Alderman, J., Collins, J. K., Lane, W., Mathewson, A. and Berney, H. (2002). Application of magnetohydrodynamic actuation to continuous flow chemistry. Lab on a Chip. 2: 224 -230.

[4] West, J., Gleeson, J. P., Alderman, J., Collins, J. K. and Berney, H. Structuring B. (2003). Laminar flows using annular magnetohydrodynamic actuation. Sensors and Actuators. 96: 190 - 199.

[5] Bestman, A. R. and Adjepong, S. N. (1988). Unsteady hydromagnetic free- convection flow with radiative heat transfer in a rotating fluid. Space Sci. 143: 73 - 80.

[6] Ghoshdastidar, P. S. Heat Transfer. Oxford University Press. UK. 2004.

[7] Peterson, J., Tuttle, N., Cooper, H. and Baukal, C. (2007). Minimize facility flaring. Hydrocarbon Processing. 111 115 .

[8] Korycki, R. (2006). Sensitivity analysis and shape optimization for transient heat conduction with radiation. Int J. Heat Mass Transfer. 49: 2033 - 2043.

[9] Gbaorun, F. K., Ikyo, B., Iyorzor, B. and Okanigbun, R. (2008). A heat model for temperature distribution in a laptop computer. J. of NAMP. 12: 201-206.

[10] Jha, B. K. (2001). Natural Convection in unsteady MHD Couette flow. Heat and Mass Transfer. 37:329-331.

[11] Ogulu, A. and Motsa, S. (2005). Radiative heat transfer to magnetohydrodynamic Couette flow with variable wall temperature. Physica Scripta. 71:336-339.

[12] Mebine, P. (2007). Radiation effects on MHD Couette flow with heat transfer between two parallel plates. Global J. Pure and Appl.Math. 3(2): 1 - 12.
[13] Jha, B. K. and Ajibade, A. O. (2009). Free convective flow of heat generating/ absorbing fluid between vertical porous plates with periodic heat input. Int. Comm. Heat and Mass Transfer. 36:624-631.

[14] Jha, B. K. and Ajibade, A. O. (2010). Unsteady free convective Couette flow of heat generating/absorbing fluid. Int. J. Energy and Tech. 2 (12):1-9.

[15] Israel-Cookey, C., Amos, E. and Nwaigwe, C. (2010). MHD oscillatory Couette flow of a radiating viscous fluid in a porous medium with periodic wall temperature. Ameri.J Sci. Indust.Res. 1(2) : 326-331

[16] Narahari, M. (2010). Effects of thermal radiation and free convection currents on the unsteady Couette flow between two vertical parallel plates with constant heat flux at one boundary. WSEAS Transactions on Heat and Mass Transfer. 5(1): 21-30.

[17] Deka, R. K. and Bhattacharya, A. (2011). Unsteady free convective Couette flow of heat generating/absorbing fluid in porous medium. Int. J. Math. Arch. 2(6):853-863

[18] Alagoa, K. D., Tay, G. and Abbey, T. M. (1999). Radiation and free convection effects on a MHD flow through a porous medium between infinite parallel plates with time-dependent suction. Astro. Space Sci. $260: 455-468$.

[19] Gbadeyan, J. A., Daniel, S. and Kefas, E. G. (2005). The radiation effect on electrohydrodynamic froth flow in vertical channel. J. Math. Associ. Nigeria. 32(2B) :388-396.

[20] Baoku, I. G., Israel-Cookey, C. and Olajuwon, B. I. (2010). Magnetic field and thermal radiation effects on steady hydromagnetic couette flow through a porous channel. Surveys in Mathematics and its Applications. 5 :215 - 228

[21] Cogley, A. C. L., Vincenti, W. G. and Gilles, E. S. (1968). Differential approximation for radiative heat transfer in a non grey gas near equilibrium. Am. Inst. Aeronat. Astronaut. J. 6: 551-553.

[22] Grief, R., Habib, I. S. and Lin, J. C. (1970). Laminar convection of a radiating gas in a vertical channel. J. Fluid Mech. 46 :513-520. 